\title{
MARS: An Educational Environment for Multiagent Robot Simulations
}

\author{
Marco Casini and Andrea Garulli \\ Dipartimento di Ingegneria dell'Informazione e Scienze Matematiche, Università degli Studi di Siena, Via Roma 56, \\ 53100 Siena, Italy
}

Correspondence should be addressed to Marco Casini; casini@ing.unisi.it

Received 15 July 2016; Accepted 2 August 2016

Academic Editor: Aiguo Song

Copyright ( 2016 M. Casini and A. Garulli. This is an open access article distributed under the Creative Commons Attribution License, which permits unrestricted use, distribution, and reproduction in any medium, provided the original work is properly cited.

\begin{abstract}
Undergraduate robotics students often find it difficult to design and validate control algorithms for teams of mobile robots. This is mainly due to two reasons. First, very rarely, educational laboratories are equipped with large teams of robots, which are usually expensive, bulky, and difficult to manage and maintain. Second, robotics simulators often require students to spend much time to learn their use and functionalities. For this purpose, a simulator of multiagent mobile robots named MARS has been developed within the Matlab environment, with the aim of helping students to simulate a wide variety of control algorithms in an easy way and without spending time for understanding a new language. Through this facility, the user is able to simulate multirobot teams performing different tasks, from cooperative to competitive ones, by using both centralized and distributed controllers. Virtual sensors are provided to simulate real devices. A graphical user interface allows students to monitor the robots behaviour through an online animation.
\end{abstract}

\section{Introduction}

It is a common opinion that experimental validation of control algorithms involving mobile robots is a mandatory practice in this field of research. Unfortunately, performing such experiments is usually costly and time demanding, especially when a large number of vehicles are involved in these experiments. When dealing with mobile robotics in education, it is often difficult to let students perform real experiments, due to several reasons like, for instance, large number of students, inadequate laboratories, lack of economic resources, and so forth. A possible solution consists in using software simulators able to accurately reproduce robot behaviours. Such simulators can be effectively used by students during the courses. Nowadays, a number of mobile robot simulators are available, developed by both commercial companies and research institutions. Examples of commercial products are Webots [1], V-rep [2], MobotSim [3], and Virtual Robotics Toolkit [4]. Moreover, many simulators have been developed in academic institutions; see [5-10], just to cite a few. These simulators provide several nice features to the user, like very accurate modelling of the mechanics of the vehicles, a detailed description of the environment, and realistic animations showing the behaviour of the robot team. On the other hand, they usually require a significant effort on the student side, in order to get acquainted with a specific programming language to implement the control algorithms for driving the robots.

In this paper, a multirobot simulator named MARS is described. Initially, this project was launched with the purpose of simulating the behaviour of mobile robots built with Lego Mindstorms bricks [11] embedded in the remote laboratory named Automatic Control Telelab (ACT); see [12-14]. Then, its scope has been extended to provide an educational easy-to-use simulator aimed at testing control algorithms for generic robots working in user-defined environments and equipped with various sensors. Unlike the above cited simulators, it does not concentrate on the dynamical model of robots and on virtual reality animation. Instead, it is oriented to rapid prototyping of the control strategies designed to accomplish the requested task. In order not to force students to learn a specific language, the simulator is based on the 
Matlab environment, which is widely employed within engineering programs. Thanks to these features, designing and validating control algorithms are much easier and faster than in other robot simulators. Several kinds of experiments can be performed in $M A R S$, from cooperative to competitive ones, involving a number of different tasks like path planning and obstacle avoidance [15], coverage, pursuer-evader games [16], rendezvous, and so forth. A key feature of MARS is the ability to design add-ons, that is, functions able to implement special features. Students may take advantage of this capability by embedding in their projects predefined add-ons or addons designed by other students. In addition, robots may be equipped with virtual sensors simulating real devices, like sonar or laser range finders. MARS has been successfully adopted in secondary school and undergraduate engineering courses at the University of Siena. Despite its simplicity, it may be also used as a research tool, allowing researchers to design and validate complex controllers before the actual implementation on real vehicles. A description of a preliminary version of the simulator architecture is sketched in [17].

The paper is organized as follows. In Section 2, a brief overview of the MARS project is summarized, while its software architecture is described in detail in Section 3. A session example is reported in Section 4, showing how the user can easily implement a controller and simulate it. Teaching experiences involving $M A R S$ carried out at the University of Siena are reported in Section 5. Experiments related to several different tasks are presented in Section 6, to demonstrate the power and flexibility of the proposed simulator. Finally, conclusions and future developments are given in Section 7.

\section{Simulator Overview}

The Multiagent Robot Simulator (MARS) (website: http:// mars.diism.unisi.it/) is a software simulator whose aim is to provide a virtual environment for simulating teams of mobile robots. Within $M A R S$, it is possible to simulate robots moving in both structured and unstructured twodimensional workspaces. Holonomic and nonholonomic vehicles equipped with different types of sensors can be considered, thus leading to heterogeneous robot teams.

Let $x_{i}(t)$ and $y_{i}(t)$ be the position of the $i$ th robot at time $t$, and let $v_{x, i}(t)$ and $v_{y, i}(t)$ be its velocities along the two coordinate axes. Holonomic robots are driven by their velocities along the two coordinate axes, so their kinematics can be easily expressed as

$$
\begin{aligned}
& \dot{x}_{i}(t)=v_{x, i}(t), \\
& \dot{y}_{i}(t)=v_{y, i}(t) .
\end{aligned}
$$

Instead, nonholonomic vehicles are implemented as unicycles, whose poses evolve as

$$
\begin{gathered}
\dot{x}_{i}(t)=v_{i}(t) \cos \left(\theta_{i}(t)\right), \\
\dot{y}_{i}(t)=v_{i}(t) \sin \left(\theta_{i}(t)\right), \\
\dot{\theta}_{i}(t)=\omega_{i}(t),
\end{gathered}
$$

where $v_{i}(t)$ and $\omega_{i}(t)$ are the linear and angular speed, respectively, and $\theta_{i}(t)$ denotes the robot orientation with

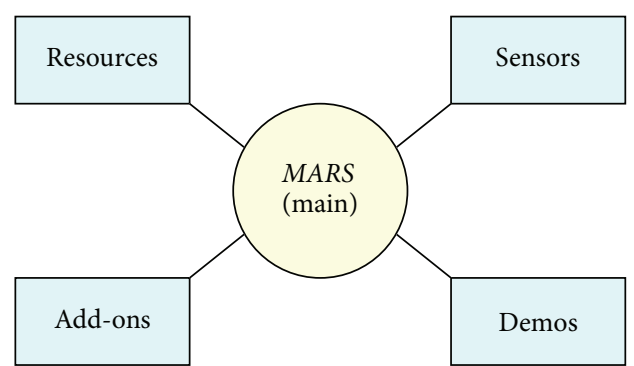

FIGURE 1: MARS software architecture.

respect to the $x$-axis. All kinematic equations are discretized for simulation. Sampling time is set by the user and robots change their status at each time step.

Robots can be equipped with the same controller or with different controllers as well, for example, for experiment involving one leader and many followers. Several types of control strategies can be implemented as follows.

Centralized Control with Full Information. It is assumed that the complete information about the environment and all robot poses is known by a supervisor controller. At each time step, such a controller is in charge of computing the desired velocities for all agents in the network.

Centralized Control with Partial Information. Unlike the previous setting, one may assume that the supervisor controller knows only partial information about robot status, like, for instance, position but not orientation.

Distributed Control with Partial Information. Each robot has to compute its own velocities based on its pose and the poses of some of the other vehicles, for instance, of its neighbors. It may also know partial information about other agents, for example, their position but not their orientation.

Distributed Control with Sensor Information. Agents have to compute their speed by means of measurements obtained by embedded virtual sensors which provide the only available information.

\section{Software Architecture}

MARS has been developed in Matlab and consists of a set of functions which can be divided into five main groups (see Figure 1).

Main File. The file called mars. $m$ represents the main function of the simulator and it is the function to be launched to run a simulation.

Resource Files. These are functions which can be used by the main file and/or by users to provide specific functionalities (e.g., check whether collisions occur).

Sensor Files. These functions allow the simulation of virtual sensors mounted on the robots, like proximity sensors, laser range finders, and so forth. 
Add-Ons Files. MARS allows one to include in the environment a bunch of features of interests, like the presence of obstacles, Voronoi diagrams, and so forth. Such functionalities are implemented through add-ons predefined within the simulator or designed by users.

Demo Files. These files are useful to show demonstrations of the simulator functionalities. They can be used as a starting point to develop new experiments.

Hereafter, the main file (mars.m) is described in detail. This is the file the user has to run to start the simulation. It consists of a Matlab function whose syntax is defined as follows:

[out_data] $=$ mars (exp_file, stop_time, speed, options)

(i) exp_file is the name of the function containing the experiment to be simulated, which has to be defined by the user. Demo files can be used as well. This is a mandatory input parameter.

(ii) stop_time specifies the time duration of the experiment in seconds.

(iii) speed denotes the playback speed of the simulation. speed $=1$ (default) means that the simulation runs at actual time; that is, the duration of the simulation is the same as that of the real experiment. Greater values can be used to increase the simulation speed, while lower values can be used to decrease it. Special values are speed $=0$ which means "manual mode"; that is, simulation proceeds at each key pressed, and speed $=$ "max" which runs the simulation at the maximum speed allowed by the available computational power.

(iv) options is a structure defining options to be used in the simulation. All the fields of the experiment status structure (Exp_status) can be set, see Table 1.

(v) out_data is a structure containing all the experiment data including robot poses, sensor outputs, and workspace features for each time step. This dataset is useful to perform offline analysis of the experiment.

All the data involving a simulation are internally stored in the experiment status structure called Exp_status. Such a structure contains data which remain constant over time, like the mechanical characteristics of the robots, as well as dynamical variables like robot poses. The main fields of Exp_status are reported in Table 1.

The body of mars. $m$ can be divided into 3 sections.

Parameter Definition. In this section, default values are assigned to parameters governing the experiment, like, for instance, the default sampling time, the workspace geometry, and the vehicles characteristics. Such parameters can be changed by the user both from the command line (setting the options parameter in the MARS call, as described above) and from the user-defined function of the experiment (exp_file).

Initialization. During this phase, the experiment file exp_file is checked and relevant information is set, like the number of

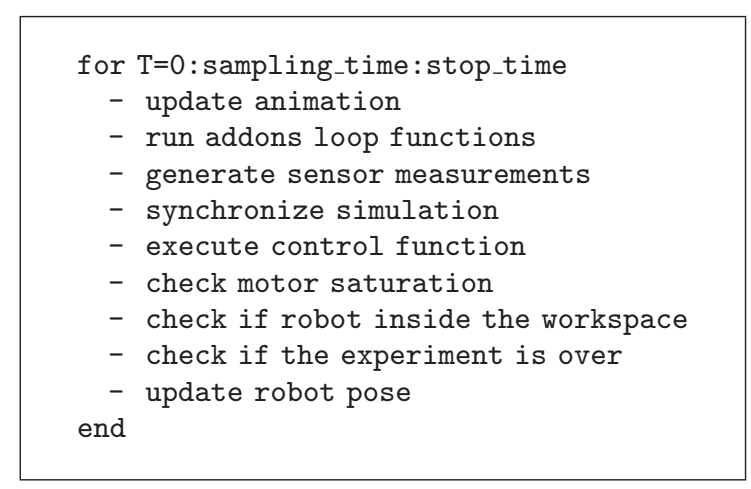

Algorithm 1: Sketch of the routines inside the main loop.

robots used, their initial conditions, the animation parameters, and the used add-ons. Moreover, experiment parameters are overwritten if specified.

Main Loop. This section is in charge of simulating the experiment and can be seen as the core of the project. A sketch of the main commands executed inside the loop is shown in Algorithm 1. The loop is repeated every sampling time and it is synchronized on the basis of the playback speed. At each time step, robot dynamics, sensor measurements, and addons routines are computed and the animation is updated. Additional computations concern collision detection and the storage of data in the output structure out_data.

On the user side, the only thing to do is to generate the experiment control file. Let us assume that exp_file. $m$ is the name of the user-defined file defining the experiment controller. This file has to adhere to the following syntax:

[Command, Exp_status] = exp_file (Exp_status, Init flag)

(i) Exp_status denotes the experiment status structure. The main structures fields are listed in Table 1.

(ii) Init_flag is a boolean variable establishing whether the simulator is in the initialization or in the simulation phase.

(iii) Command is a matrix containing the desired input speeds of all the robots at the current time step.

The task of the user can be summarized as follows. By exploiting the data contained in Exp_status, design a control law for each robot which returns a desired robot speed. For holonomic robots, exp_file has to generate the velocities $v_{x, i}(t), v_{y, i}(t)$ along the two cartesian axes, while for nonholonomic robots it has to return the linear and angular velocities, $v_{i}(t)$ and $\omega_{i}(t)$.

Therefore, to sum up, the user has just to freely write the code of the desired control law generating the desired velocities and finally pack them up within the matrix Command with the following structure:

(i) Holonomic robots: Command $=\left[\begin{array}{llll}v_{x, 1} & v_{x, 2} & \cdots & v_{x, n} \\ v_{y, 1} & v_{y, 2} & \cdots & v_{y, n}\end{array}\right]$

(ii) Nonholonomic robots: Command $=\left[\begin{array}{cccc}v_{1} & v_{2} & \ldots & v_{n} \\ \omega_{1} & \omega_{2} & \ldots & \omega_{n}\end{array}\right]$ 
TABLE 1: Description of main Exp_status fields.

\begin{tabular}{|c|c|}
\hline Field name & Description \\
\hline Robots & Number of robots used (default $=1)$ \\
\hline Pose & Robots pose $[x$ (meters); $y$ (meters); angle (radians)] \\
\hline Initial_pose & Initial pose of robots \\
\hline Sampling_time & Sampling time (seconds) \\
\hline Iteration & Actual iteration of the main simulation loop \\
\hline Time & Elapsed experiment time (seconds) \\
\hline Stop_time & Max duration of the experiment (seconds) \\
\hline Workspace & Vertexes defining the polygonal workspace in clockwise order \\
\hline Bounds & Bounds of the outer box of the workspace (meters) $\left[\min \_x, \max \_x, \min _{-} y, \max _{-} y\right]$ \\
\hline Non-holonomic & True if robots have nonholonomic drive; false for holonomic drive $($ default $=$ true $)$ \\
\hline Command_delay & Transmission delay (seconds) \\
\hline Geometric_center & Coordinates of the geometric center of the robot \\
\hline Exp_over & True if the experiment is over \\
\hline Exp_over_msg & Message describing why the experiment is over \\
\hline Filename & Name of the controller file \\
\hline Version & Simulator version \\
\hline Addons & List of add-ons to be used \\
\hline Addons_suffix & List of suffixes to be used in add-on's filename \\
\hline Robot & Robot mechanical characteristics \\
\hline Robot.Max_linear_speed & Maximum linear speed (m/s) \\
\hline Robot.Wheels_semiaxis_length & Semiaxis length $(\mathrm{m})$ \\
\hline Robot.Diameter & Diameter of robot chassis $(\mathrm{m})$ \\
\hline Robot.Distance_center_barycenter & Distance from the geometric center of the robot to the center of rotation \\
\hline Animation & Animation structure \\
\hline Animation.Title & Title of the simulation (default = "[Simulation]") \\
\hline Animation.Grid & Enable/disable grid $($ default $=$ true $)$ \\
\hline Animation.Show_initial_pose & Show/hide initial pose of robots $($ default $=$ true $)$ \\
\hline Animation.Show_real_shape & If true, the actual robot shape is shown; otherwise, a pointwise robot is depicted (default $=$ true) \\
\hline Animation.Wake & Enable/disable robots' wake $($ default $=$ true $)$ \\
\hline Animation.Wake_style & $\begin{array}{l}\text { If wake is enabled, use the defined style; styles are the same as in the Matlab function plot } \\
\text { (default = "-") }\end{array}$ \\
\hline Animation.Playback_speed & Simulation playback speed in seconds (default $=1$ ) \\
\hline Animation.Enable & Enable the animation $($ default $=$ true $)$ \\
\hline Animation.Base_colors & Base colors to be used in the animation; they will be repeated if needed \\
\hline Animation.Colors & Robot colors to be used in the animation; they are computed repeating the base colors if needed \\
\hline History & Historical data structure \\
\hline History.Time & Simulation time \\
\hline History.Pose & Robot pose \\
\hline History.Command & Robot command \\
\hline History.Command_time & Time when the command is received \\
\hline
\end{tabular}

where $n$ is the number of robots, specified by Exp_status. Robots.

\section{Session Description}

In this section, an illustrative session example is reported along with the Matlab code needed to implement it. As described in the previous section, the user has to provide a control file (Example_random.m in this session example) which generates the desired robot velocities at each time sample.

The aim of this experiment is to drive two robots in a squared environment of $10 \times 10$ meters. Robots are equipped with proximity sensors (e.g., a ring of sonar) which return 


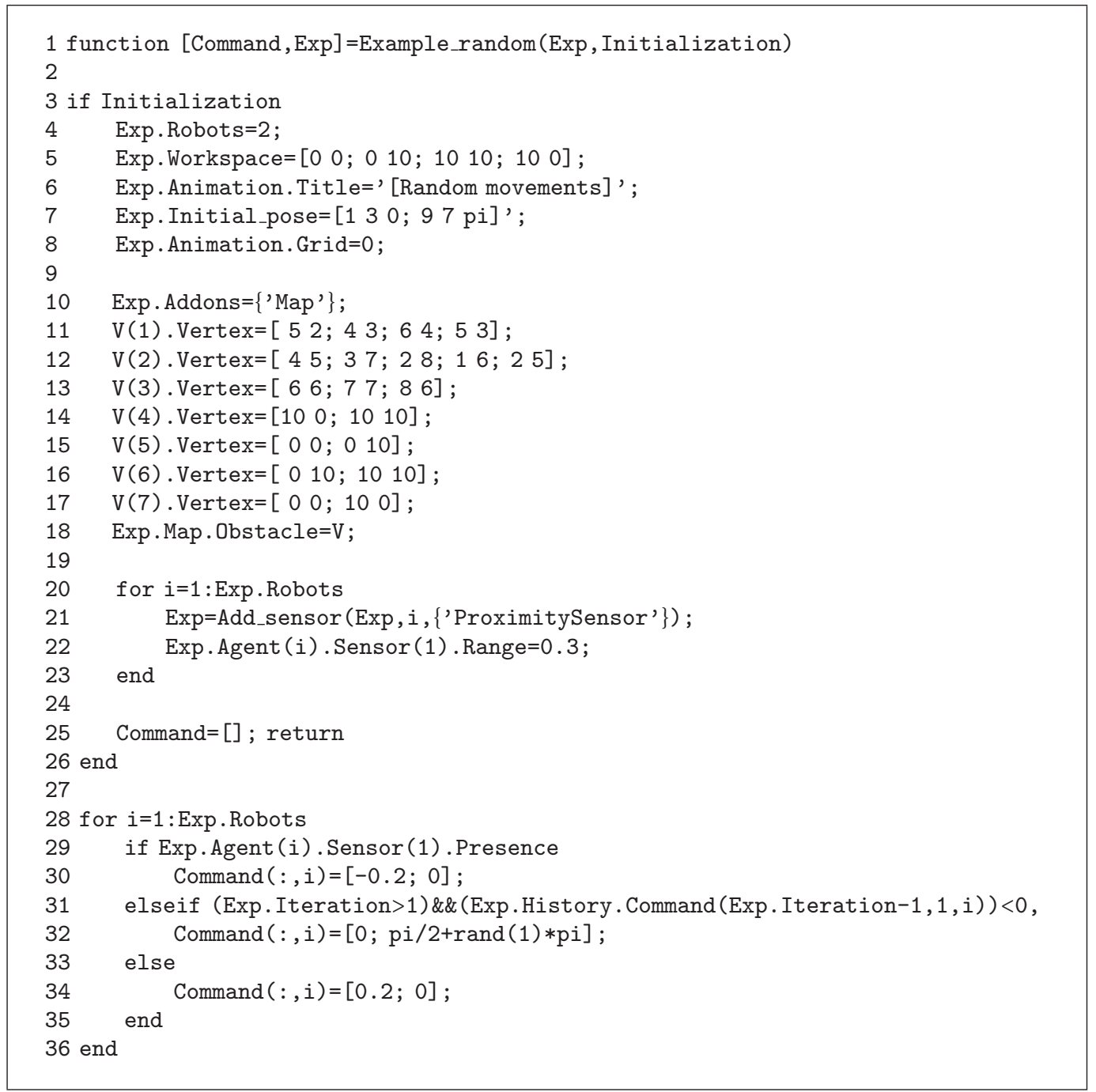

Algorithm 2: Matlab code implementing the illustrative example of Section 4.

true if an obstacle or a robot is closer than a prescribed range ( $0.3 \mathrm{~m}$ in this example). The linear speed of both robots is set as $0.2 \mathrm{~m} / \mathrm{s}$, while the angular speed is set as 0 if no obstacle is detected. Whenever a robot detects an obstacle or the other robot, it goes backward for one time step and then it rotates with a random angle. The sampling time is set as 1 second.

The overall Matlab code implementing the required task is reported in Algorithm 2, while the simulation output after $1000 \mathrm{~s}$ is depicted in Figure 2. The simulation is run by the following command: out_data = mars("Example_random", 1000, “max").

\section{Initialization (Lines 3-26)}

Lines 4-8. Several parameters are defined, including the number of robots used in the experiment, the workspace shape and size, and the initial poses of the vehicles.

Line 10. The add-on Map is loaded. It generates the new substructure Exp.Map containing all the information about the map.
Lines 11-18. Obstacles are defined through their vertices. In lines 14-18, "fictitious" obstacles are defined at the workspace boundary to prevent robots from exiting the environment.

Lines 20-23. In line 21, each robot is equipped with a proximity sensor, while in line 22 it is set to detect obstacles around a circle with radius $0.3 \mathrm{~m}$.

Line 25. It includes a mandatory code at the end of the initialization section.

Body (Lines 28-36)

Lines 29-30. For each robot, line 29 checks whether the sensor detects the presence of an obstacle or a robot inside its field of view. If a presence is found, the robot is asked to go backward with a linear velocity of $0.2 \mathrm{~m} / \mathrm{s}$ and an angular speed of 0 . Otherwise, condition in 31 is evaluated.

Lines 31-35. Condition in line 31 checks whether the linear speed at the previous time step was negative 


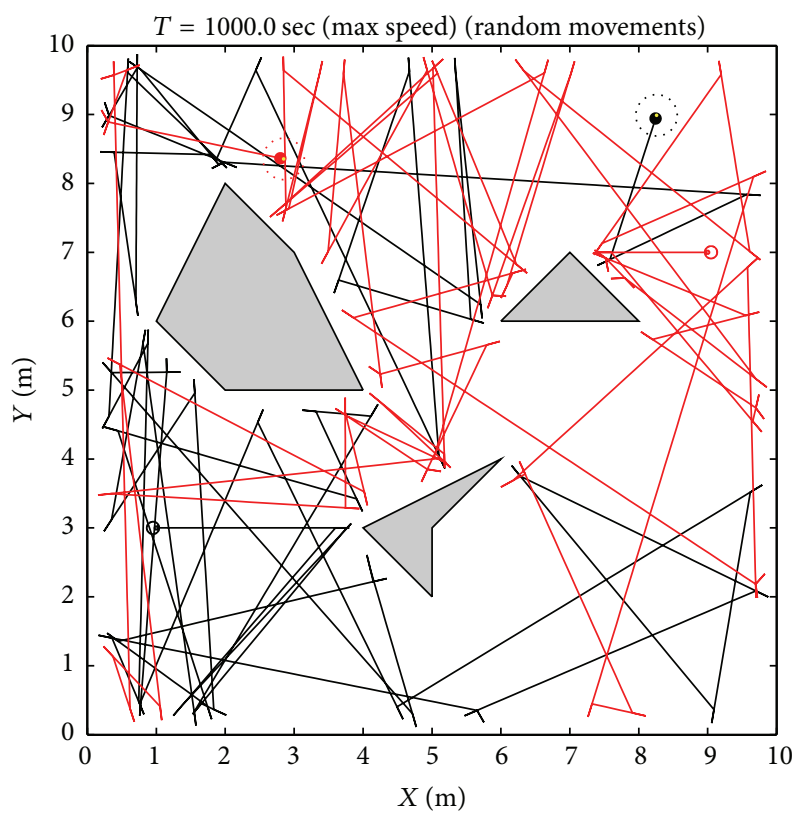

FIGURE 2: Illustrative example of two robots moving randomly in an environment with obstacles.

(and the iteration number is greater than one to prevent errors). If so, the robot stops and turns with a random angle between $\pi / 2$ and $3 / 2 \pi$; otherwise, it proceeds straight.

\section{Teaching Experiences}

Two types of educational experiences based on MARS have been assessed in the recent past, involving secondary school and bachelor students, respectively.

5.1. Secondary School Student Experiences. This experience regards 31 secondary school students who came to the university for a short stage, with the aim of stimulating their creativity and passion towards engineering topics. This activity dealt with a pursuer-evader game with one pursuer and one evader. The aim of the pursuer is to "catch" the evader, that is, to collide with it, while the evader should avoid collision. After a given time, if no collision occurs, the evader wins; otherwise, it loses. Students were asked to gather in small groups and design a control law for the evader agent. They knew the pursuer strategy which consists in going towards the evader at each time step. The evader speed was initially set as twice the pursuer speed. Then, the latter was gradually increased to make the game more challenging.

Several different control algorithms were devised by various groups. An effective solution relates to a strategy which alternatively drives the evader to one of the four corners of the rectangular workspace depending on the pursuer position (Figure 3). Such an algorithm allows the evader to escape from the pursuer when the speed ratio between the pursuer and the evader is up to 0.9. Of course, different initial positions led to different results. Moreover, it

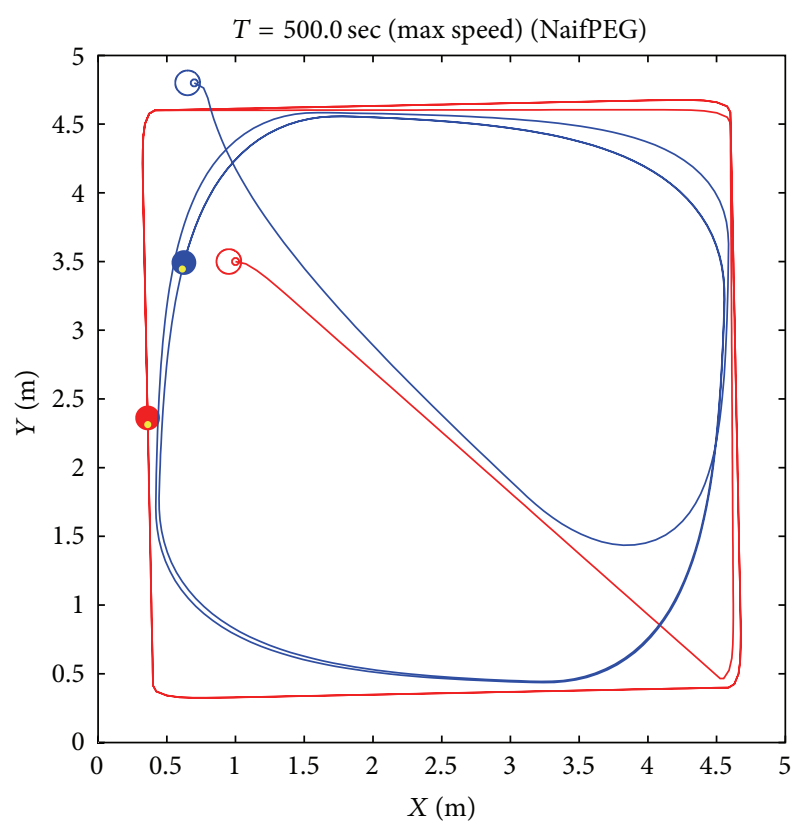

FIgURE 3: Pursuer-evader game, evader controller implemented by secondary school students. Robot poses after 500 seconds. Pursuer: blue; evader: red.

was shown to students that a slower pursuer is still able to capture the evader when using more clever strategies.

5.2. Undergraduate Student Experiences. Another educational activity which makes use of $M A R S$ is related to specific projects carried out by bachelor students, who were asked to implement control algorithms available in the literature, performing a number of different tasks. Only one student is usually involved in a given assignment. Some of these experiences are reported in the next session, concerning tasks such as coverage, pursuer-evader games, cyclic pursuit, collective circular motion, and path planning.

\section{Session Examples}

In this section, five simulation experiments are described to illustrate the features and capabilities of the proposed simulator. All the controllers have been implemented by bachelor students as a part of their final exam project. In all the experiences, robots are assumed to be nonholonomic drive vehicles.

6.1. Coverage. Coverage tasks for multiagent systems consist in arranging them to optimally cover a given environment, that is, to place them in suitable positions in order to minimize a given cost function. A convex environment is assumed and a density function representing the importance of different areas is given.

An undergraduate student was asked to implement the distributed control strategy described in [18]. Such a control law is an extension of the Lloyd algorithm [19] and it is based on the central Voronoi partitions of the environment [20]. 


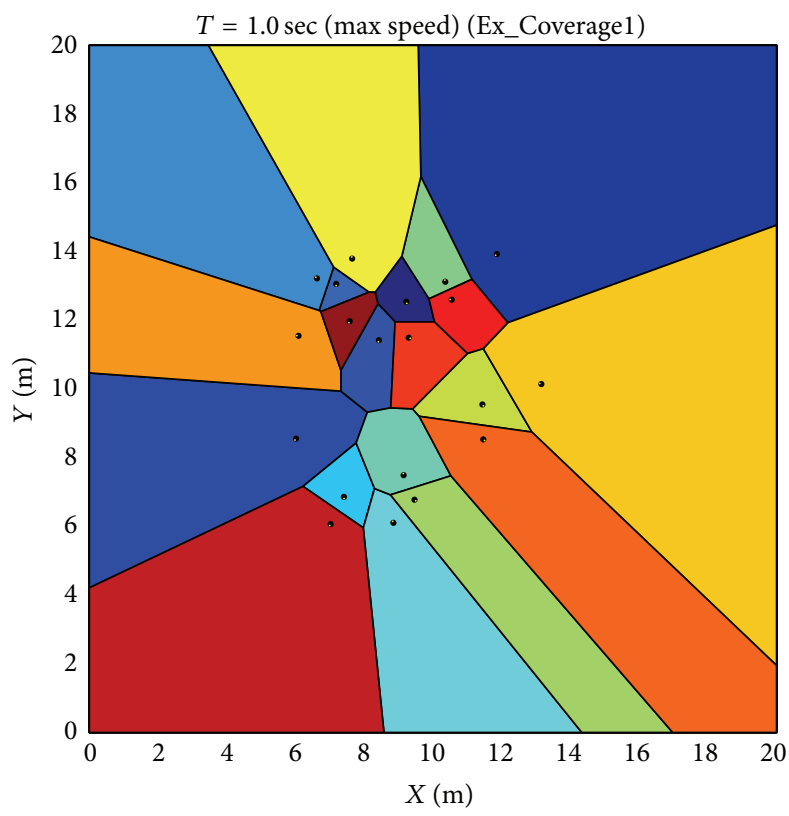

(a)

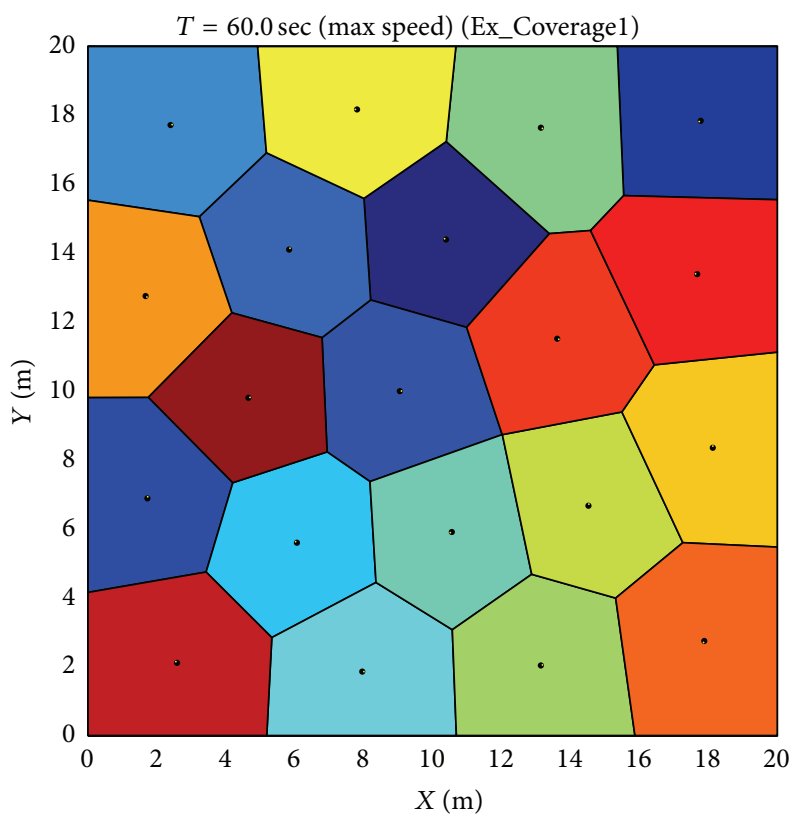

(c)

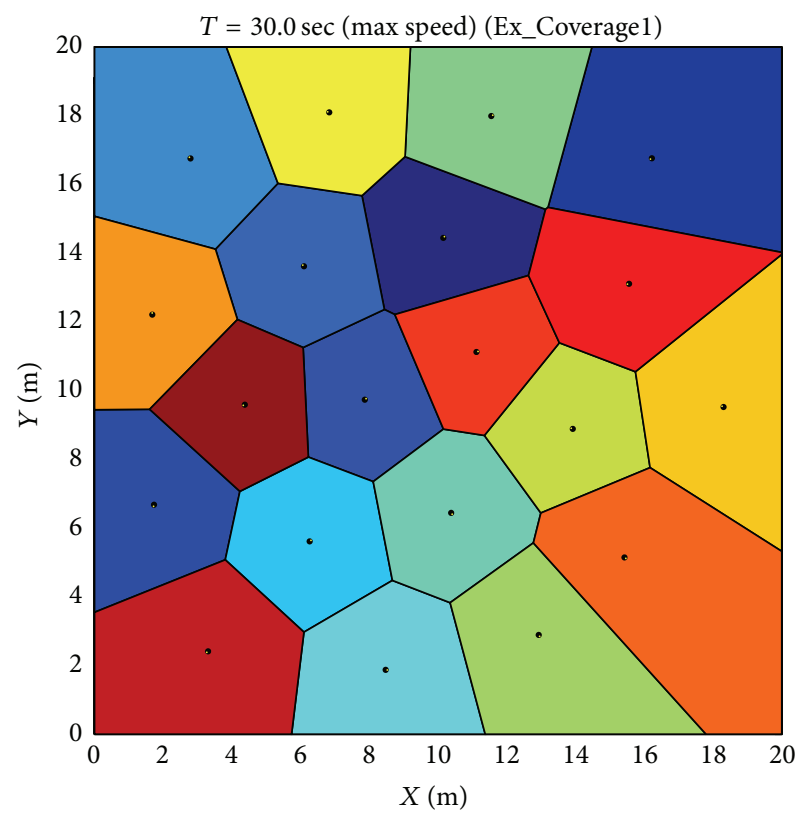

(b)

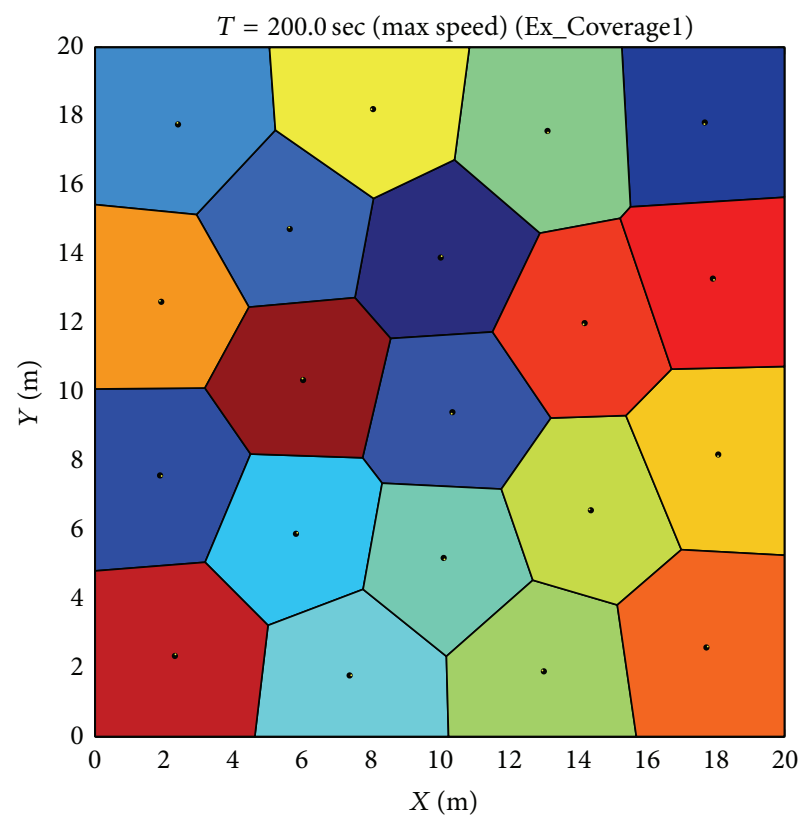

(d)

FIgURE 4: Coverage Example 1. Voronoi cells assigned to each robot for different times. (a) $t=1 \mathrm{~s}$. (b) $t=30 \mathrm{~s}$. (c) $t=60 \mathrm{~s}$. (d) $t=200 \mathrm{~s}$.

Therefore, Voronoi cells and their corresponding centroids were computed by means of the Voronoi add-on in MARS.

As a first example, an experiment involving 20 robots, uniform density, and square workspace has been performed, whose simulation results are reported in Figure 4. After $t=60$ seconds, robots almost reached their steady state position.

Since the implemented algorithm does not guarantee attaining the global minimum of the performance cost, it may happen that slightly different initial positions give rise to quite different final ones. Such a behaviour is shown in Figure 5 for 8 robots moving in a circular environment, using the same control strategy described above. In particular, the initial positions of the two experiments differ only for the position of one robot for $0.1 \mathrm{~m}$. This experience helps students to get acquainted with the key concepts like nonconvex optimization and sensitivity to initial conditions.

6.2. Pursuer-Evader Game. A pursuer-evader game with one pursuer and one evader is considered. The aim is to implement the pursuer strategy reported in [21]. Let us name this control law VoronoiPEG. Such a strategy is based on the Voronoi partition of the workspace and aims at increasing 


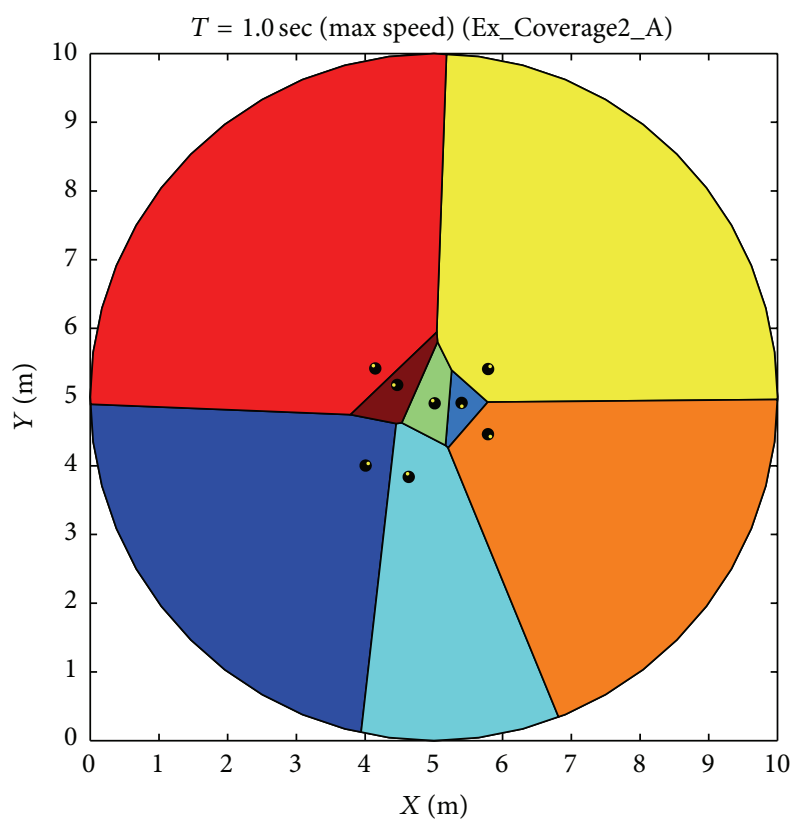

(a)

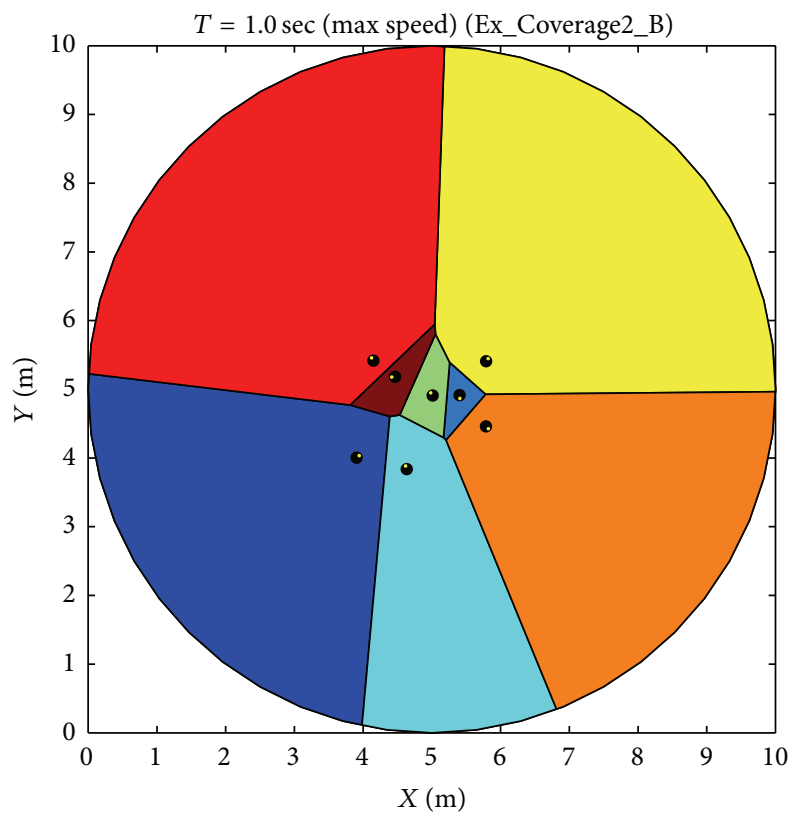

(c)

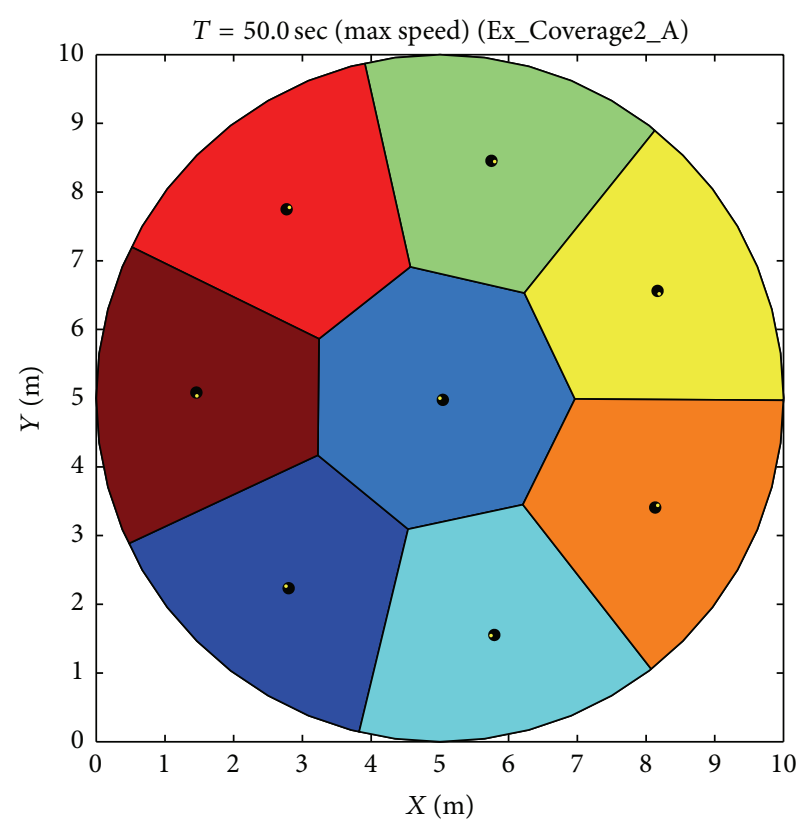

(b)

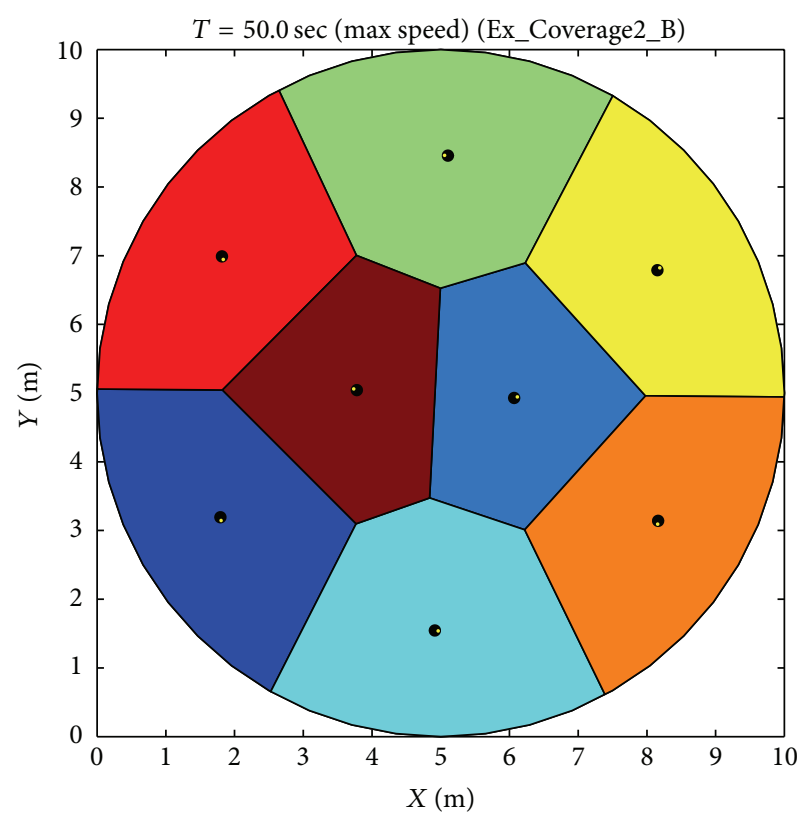

(d)

FIGURE 5: Coverage Example 2. Voronoi cells assigned to each robot for different times. (a) Initial configuration experiment A ( $t=1 \mathrm{~s}$ ). (b) Final configuration experiment A $(t=50 \mathrm{~s})$. (c) Initial configuration experiment B $(t=1 \mathrm{~s})$. (d) Final configuration experiment B $(t=50 \mathrm{~s})$.

the area of the Voronoi cell associated with the pursuer at each time instant. In [21], it is proved that the area of the Voronoi cell associated with the pursuer is increasing at each time step under any admissible evader strategy. Eventually, the evader cell reduces to zero with the consequent capture by the pursuer.

In the reported example, it is assumed that the speed of the pursuer is set as $80 \%$ of the speed of the evader. The evader will move alternatively to four points near the corners of the workspace, depending on the current position of the pursuer. The initial condition is shown in Figure 6(a). Empty and filled circles denote the initial and final poses of the robots, respectively. Robot paths are denoted by solid lines, while the light red area denotes the Voronoi cell associated with the evader (red circle). Figures 6(b) and 6(c) show the path of the two vehicles at different times. In Figure 6(d), the final configuration of the robots is displayed, showing the capture of the evader by the pursuer. 


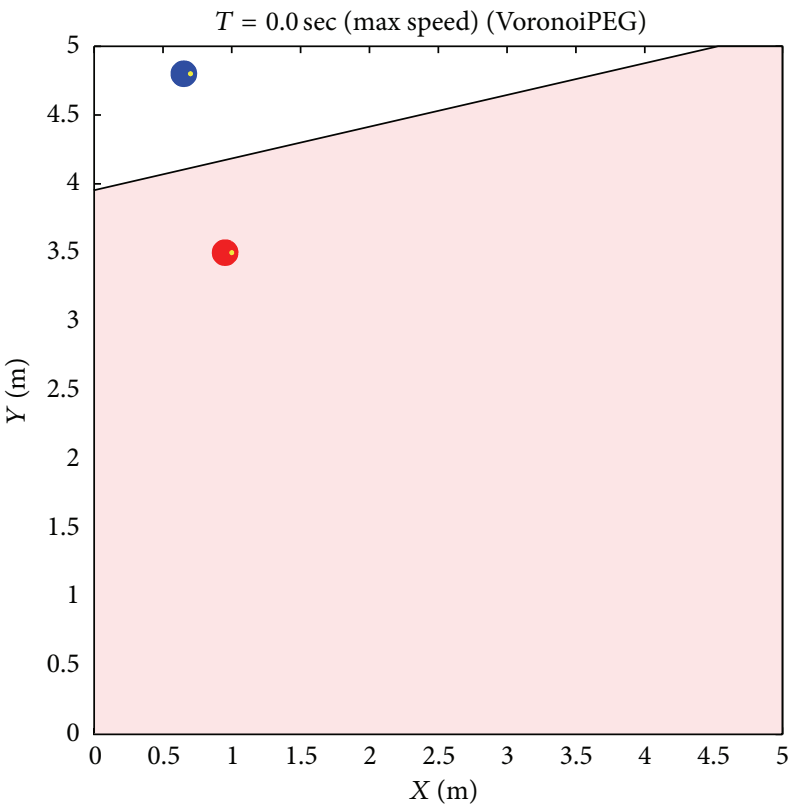

(a)

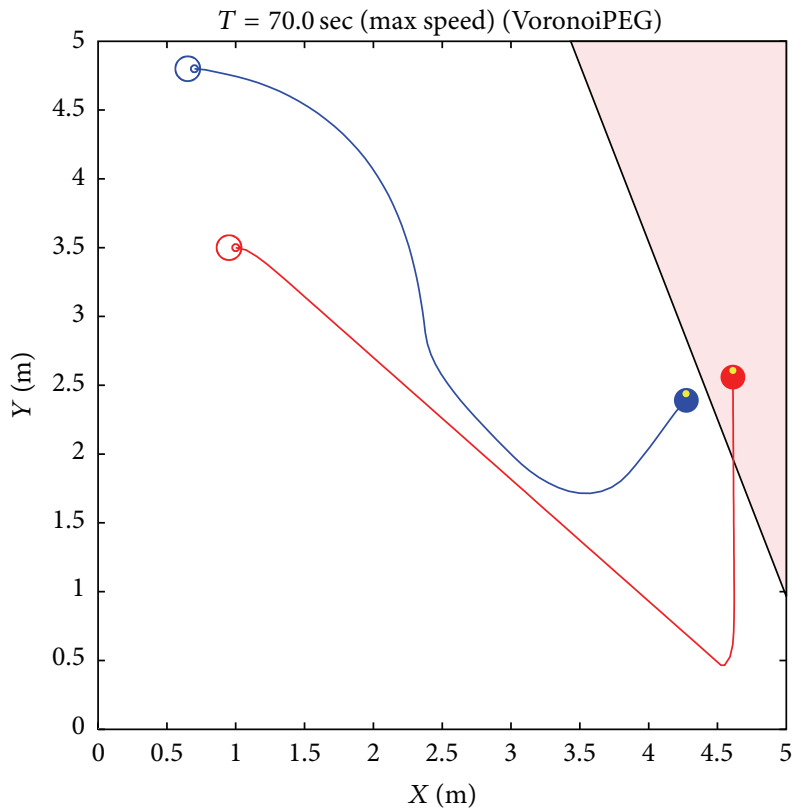

(c)

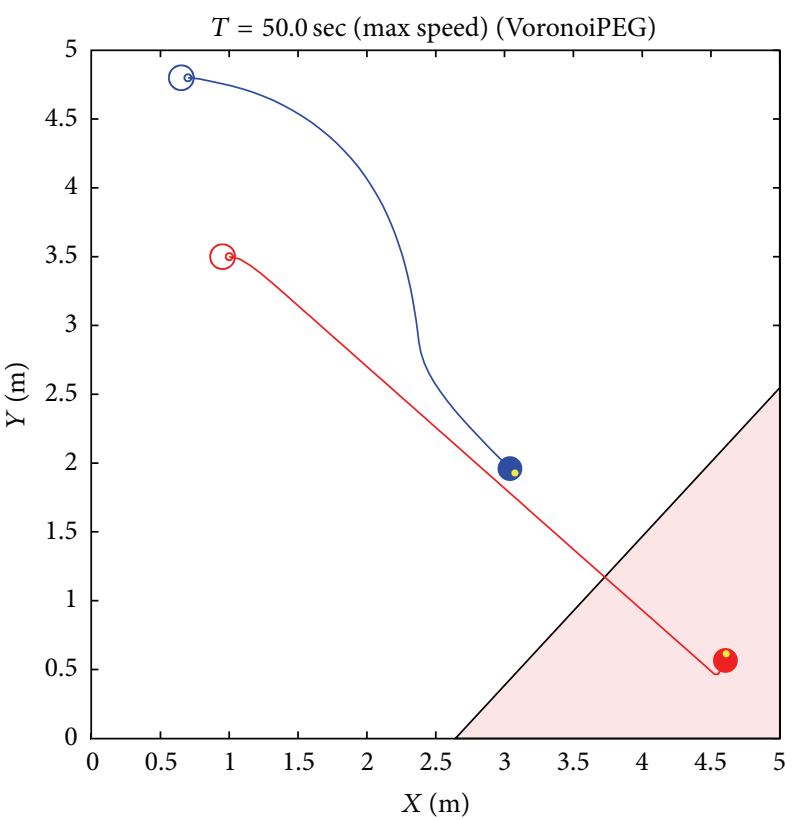

(b)

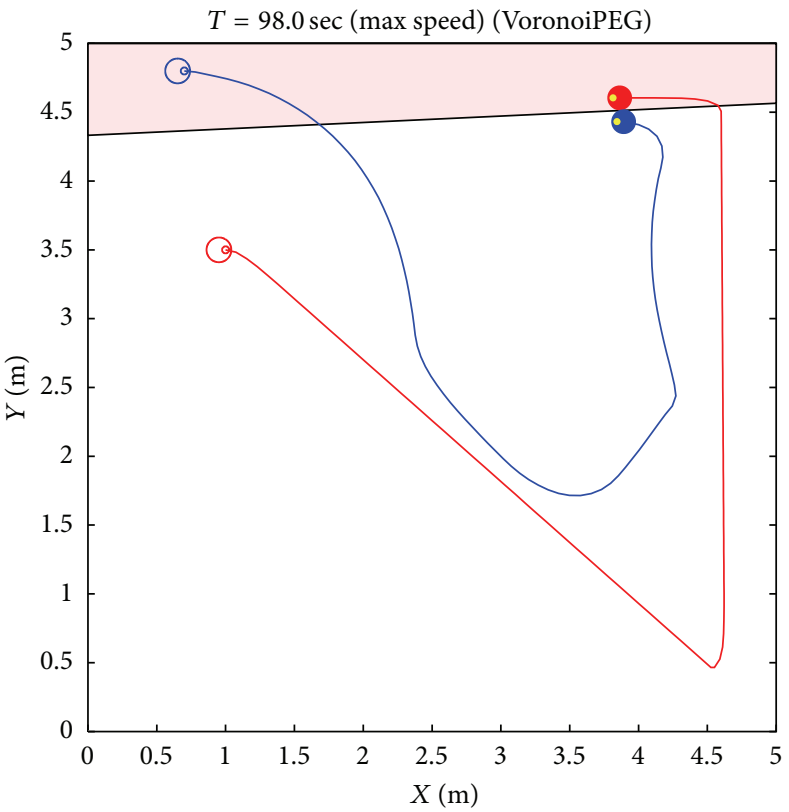

(d)

Figure 6: Pursuer-evader game example. Pose of each robot for different times. (a) Initial robot poses. (b) $t=50 \mathrm{~s}$. (c) $t=70 \mathrm{~s}$. (d) Final robot poses $(t=98 \mathrm{~s})$. Pursuer: blue; evader: red; evader Voronoi cell: light red.

6.3. Cyclic Pursuit. Cyclic pursuit is a well studied problem in formation control. In this experiment, six vehicles are assumed to be distinguishable and labeled from 1 to 6 . Each agent has to pursue the next one; that is, robot $i$ has to follow robot $i+1$ (modulo 6). It is assumed that each robot is equipped with an ideal sensor which allows it to detect the position and orientation of the robot it has to follow. The aim is to design a control algorithm driving each robot, stabilizing the platoon to regular formations. In this experiment, the decentralized control law described in [22] has been implemented, with the aim of driving vehicles around a circular trajectory. Each robot independently computes its linear and angular speed, proportional to the distance and the orientation of its preceding neighbor. With a suitable choice of the controller gains, a circular formation with equally spaced vehicles is eventually achieved, as depicted in Figure 7. This experience helps the student to understand the role of the controller parameters and to analyze how the formation scales with the number of robots in the team. 


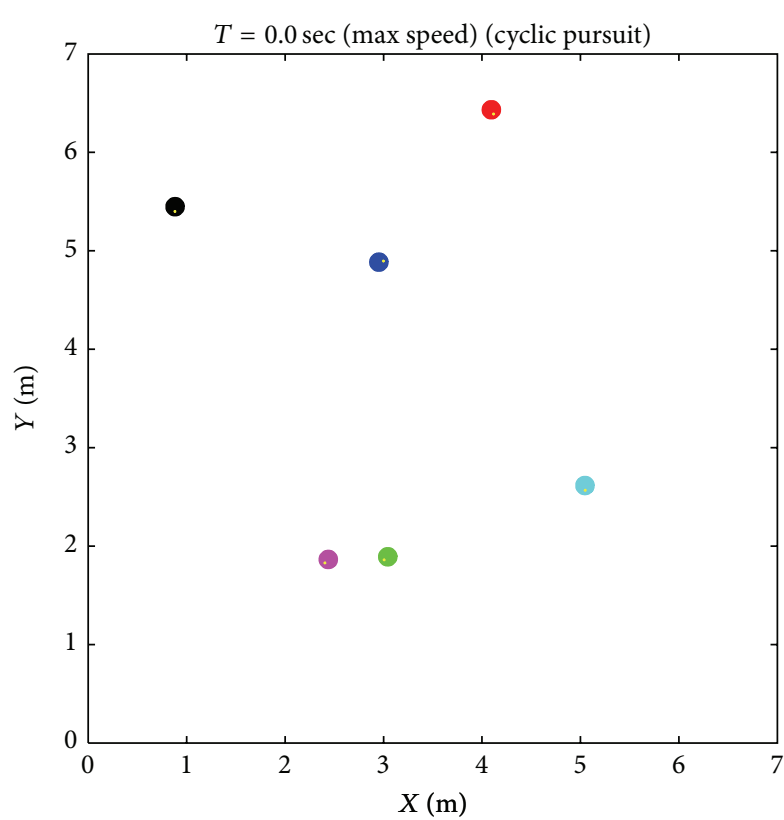

(a)

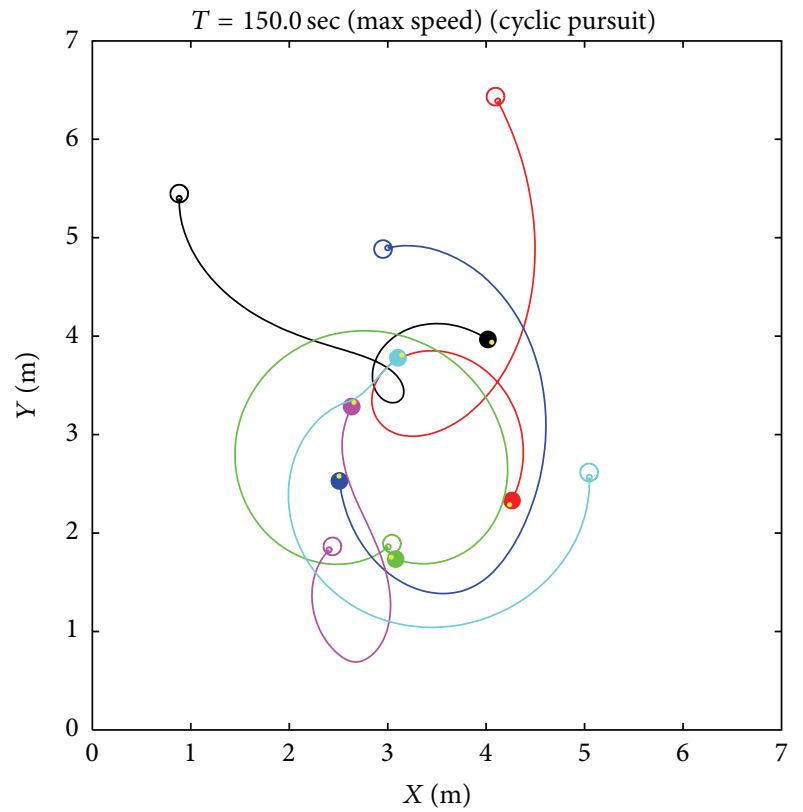

(c)

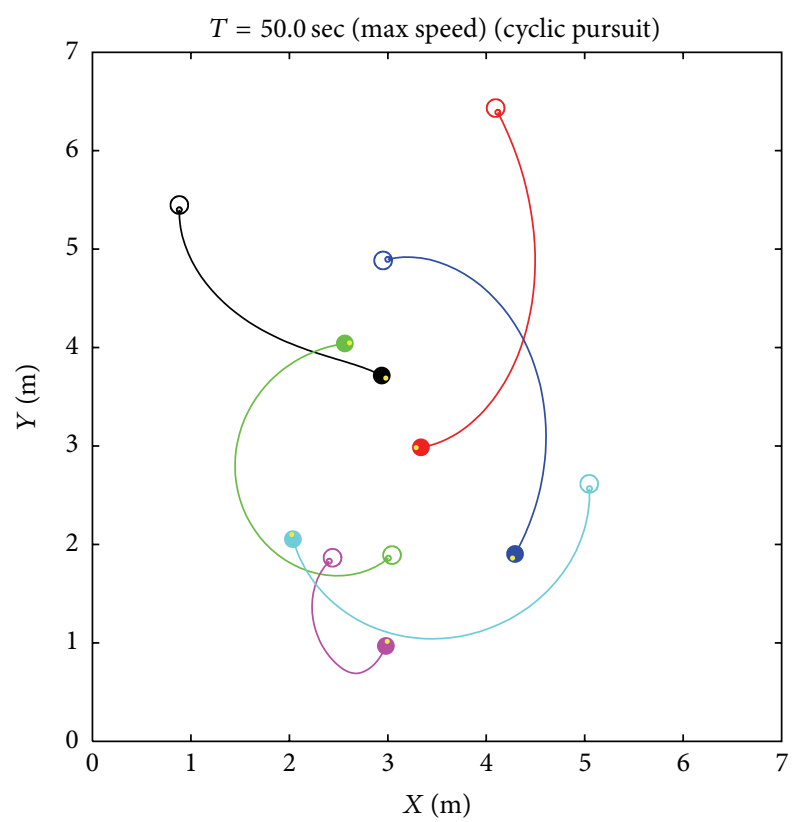

(b)

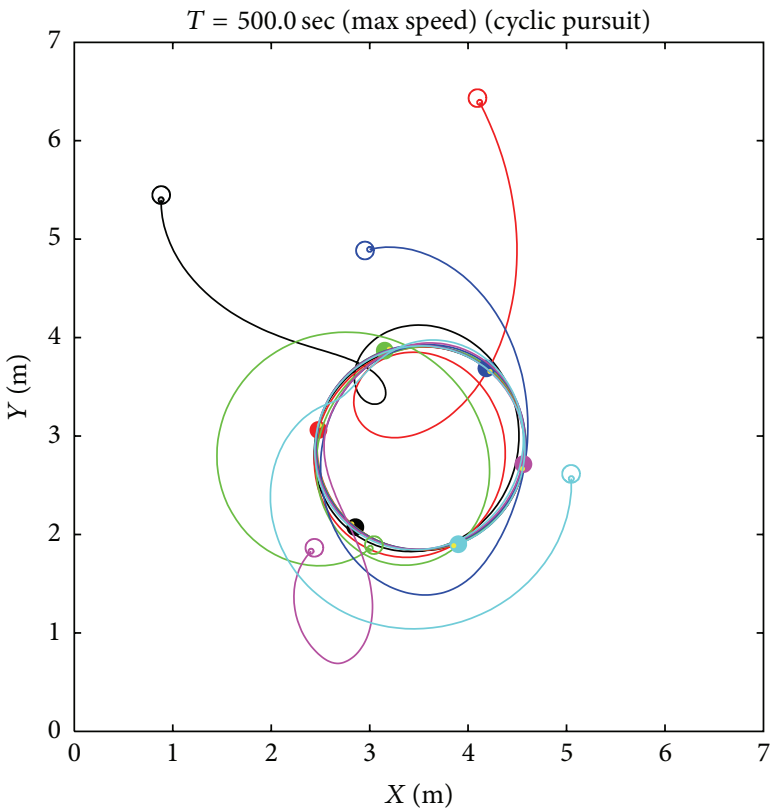

(d)

FIgure 7: Cyclic pursuit example. Pose of each robot for different times. (a) Initial robot poses. (b) $t=50 \mathrm{~s}$. (c) $t=150 \mathrm{~s}$. (d) $t=500 \mathrm{~s}$.

6.4. Collective Circular Motion. This experiment regards collective circular motion of a team of mobile robots [23, 24]. Being different from the previous experiment, here, the platoon is assumed to be composed of indistinguishable vehicles. Each robot is equipped with a range finder sensor able to provide range and bearing measurements of vehicles falling inside a given range and a proximity sensor (e.g., a sonar ring) which returns the presence of the closest vehicle.
The aim of the platoon is to encircle a moving beacon while maintaining a minimum distance among the vehicles and avoiding collisions. The distributed control law devised in [23] has been implemented. An experiment involving four robots pursuing a slow moving beacon is reported in Figure 8. Here, the beacon moves from $[3.6,2.5]^{\prime}$ to $[1.3,1.3]^{\prime}$ following a straight line. The platoon is able to pursue the moving beacon and to stabilize around a circle as soon as 


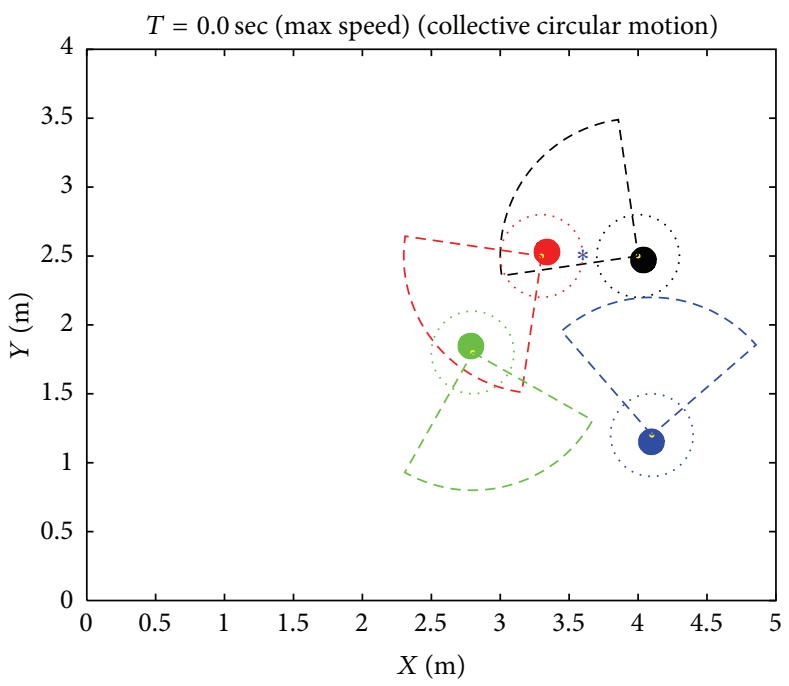

(a)

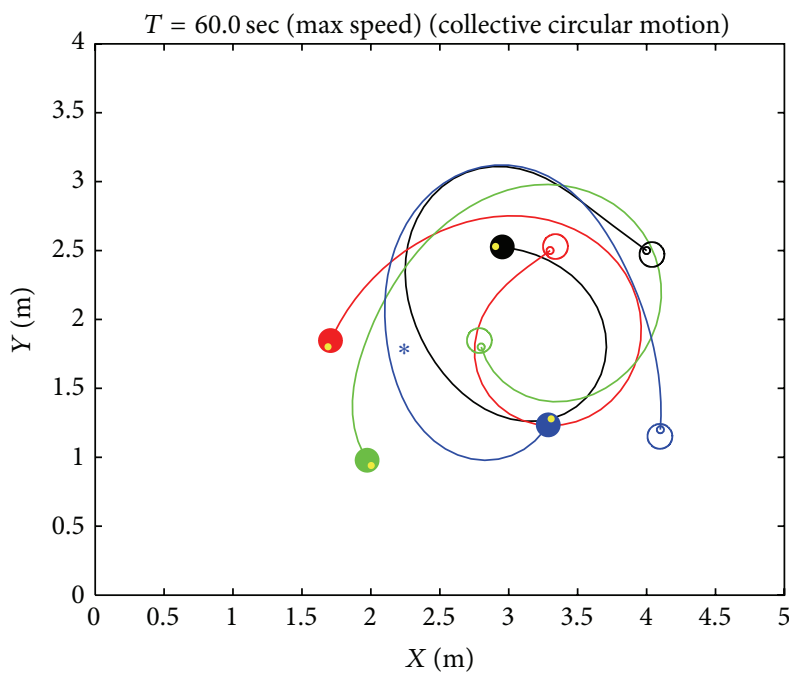

(c)

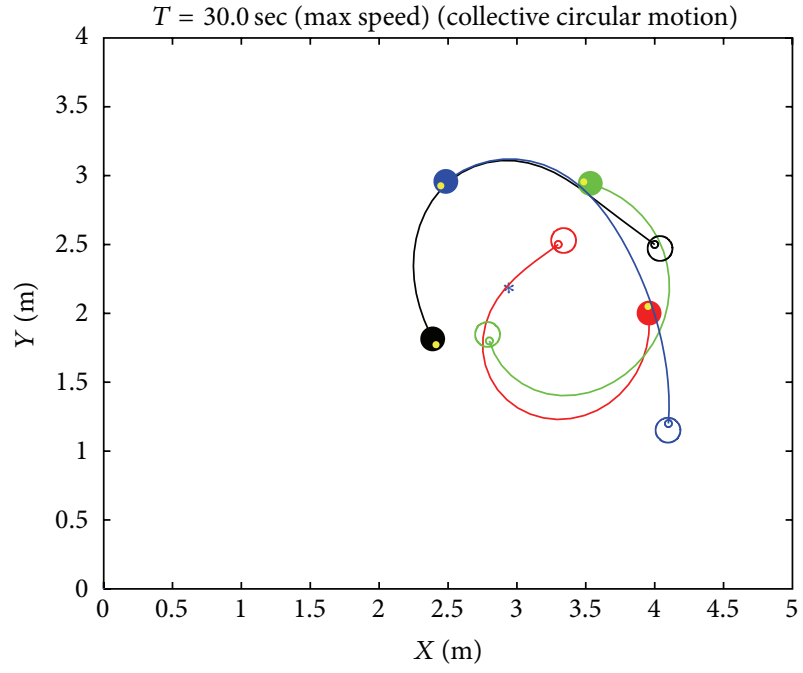

(b)

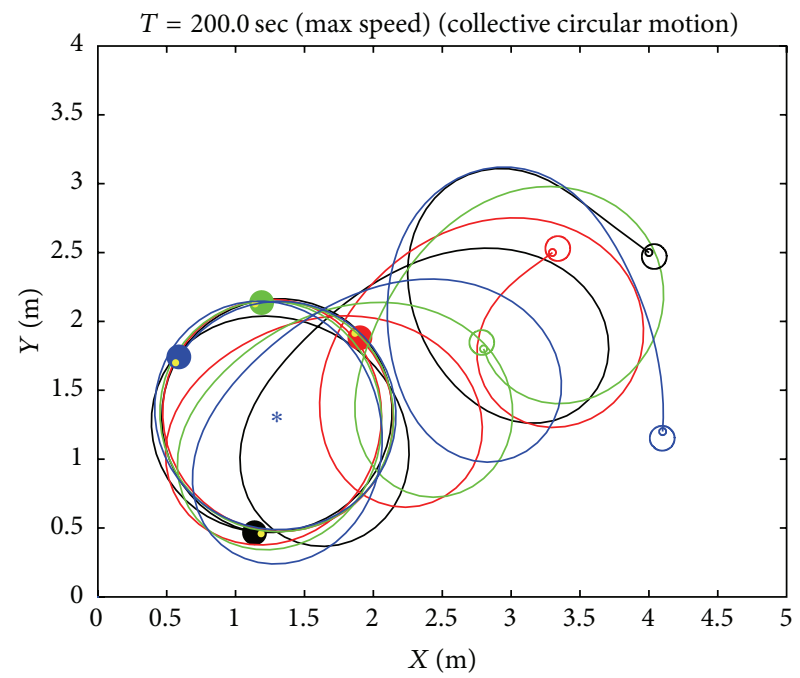

(d)

FIGURE 8: Collective circular example. Pose of each robot for different times. (a) Initial robot poses and sensor field of view. (b) $t=30 \mathrm{~s}$. (c) $t=60 \mathrm{~s}$. (d) $t=200 \mathrm{~s}$. Robots are denoted by circles while the beacon is denoted by the asterisk.

the reference stops. Also, in this case, the student can easily challenge the considered technique by changing the sensor range, initial configuration, or the beacon trajectory.

\subsection{Path Planning in Complex Environment. To demonstrate} the capabilities of MARS in managing complex environments, in Figure 9, three robots navigating inside the second floor of the authors' department are shown. The map has been generated in the same way as in the much simpler example in Algorithm 2, after extracting the vertices of each element in the environment from a CAD map. In the experiment, robots are driven by an algorithm based on the artificial potential field; see [25] for details. They are asked to avoid collisions, navigate towards a sequence of waypoints, and stop when the last waypoint is reached. Notice that while two robots are able to reach their final destination, the red one gets stuck inside a room. This behaviour is in accordance with a known issue of controllers based on the artificial potential field method, and students may easily check it. As a possible solution, waypoints should be rearranged or a new waypoint should be added.

\section{Conclusions}

In this paper, MARS, a Matlab-based simulator for mobile robotics, has been introduced. Such a tool is able to simulate teams of autonomous vehicles performing different tasks. A number of control strategies may be easily implemented, exploiting partial or full information or using data acquired by virtual sensors. In addition, users can design new add-ons and sensors to customize the simulator to specific needs.

The main purpose of this project is educational and it has been used in control systems and robotics courses at the University of Siena. The scope of this facility is not only confined to the educational one, since it can also be used 


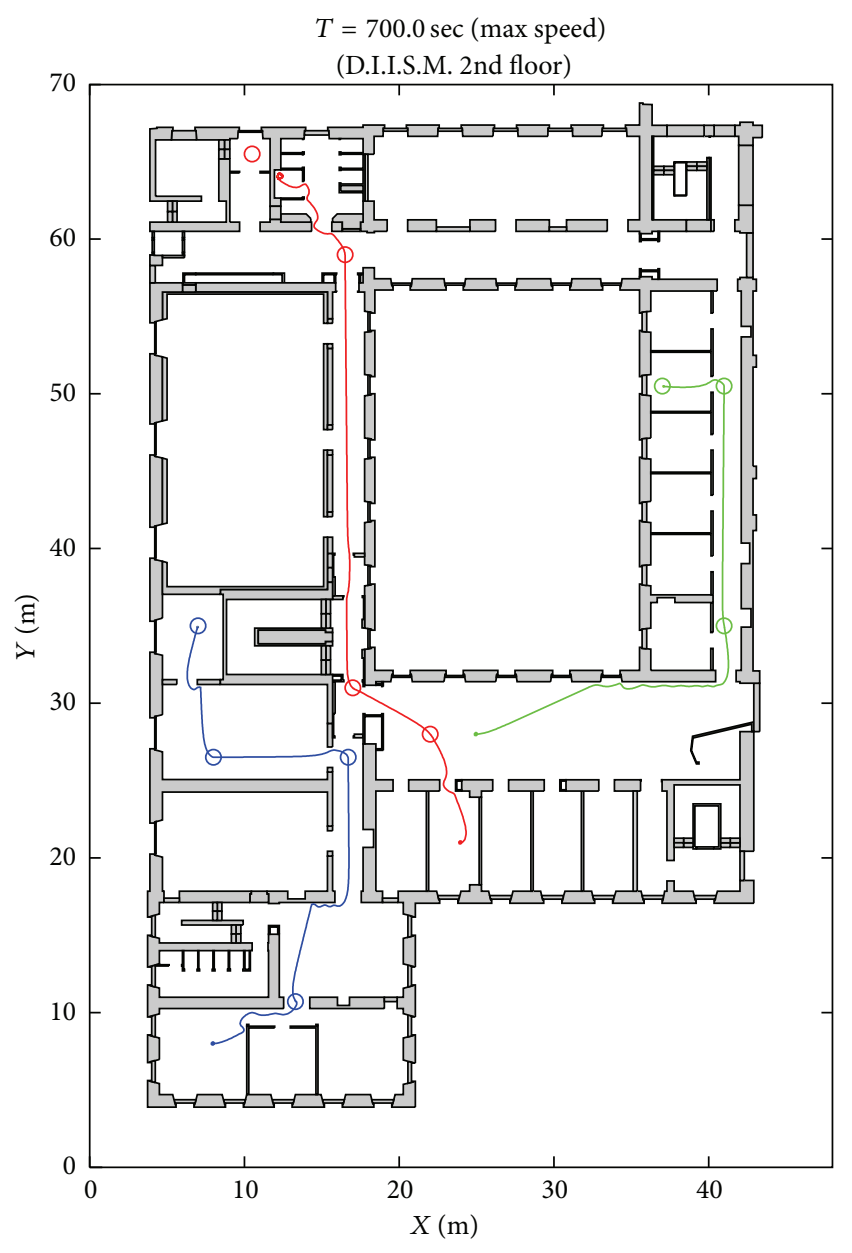

FIGURE 9: Path planning example. Three robots moving in a complex environment through a sequence of waypoints (circles).

for rapid prototyping of new multiagent control algorithms before testing them on real setups.

The MARS project is still under development and several extensions are planned for the near future, like the development of a graphical interface for defining virtual obstacles, the addition of further virtual sensors, and the simulation of communication protocols between robots. This will lead to a more realistic analysis of complex multiagent systems, such as asynchronous moving sensor networks or teams of heterogeneous mobile robots.

\section{Competing Interests}

The authors declare that there are no competing interests regarding the publication of this paper.

\section{References}

[1] Cyberbotics Ltd, Webots robot simulator, 2016, https://www .cyberbotics.com/.

[2] M. Freese, S. Singh, F. Ozaki, and N. Matsuhira, "Virtual robot experimentation platform V-REP: a versatile 3D robot simulator," in Simulation, Modeling, and Programming for Autonomous
Robots, N. Ando, S. Balakirsky, T. Hemker, M. Reggiani, and O. von Stryk, Eds., vol. 6472 of Lecture Notes in Computer Science, pp. 51-62, Springer, Berlin, Germany, 2010.

[3] Mobotsoft. MobotSim-Mobile Robot Simulator, 2016, http:// www.mobotsoft.com/?page id=9.

[4] Cogmation Robotics, Virtual Robotics Toolkit, 2016, https:// www.virtualroboticstoolkit.com.

[5] N. Koenig and A. Howard, "Design and use paradigms for Gazebo, an open-source multi-robot simulator," in Proceedings of the IEEE/RSJ International Conference on Intelligent Robots and Systems (IROS '04), pp. 2149-2154, October 2004.

[6] A. Koestler and T. Bräunl, "Mobile robot simulation with realistic error models," in Proceedings of the International Conference on Autonomous Robots and Agents (ICARA '04), vol. 1, pp. 4651, 2004.

[7] S. Carpin, M. Lewis, J. Wang, S. Balakirsky, and C. Scrapper, "USARSim: a robot simulator for research and education," in Proceedings of the IEEE International Conference on Robotics and Automation (ICRA '07), pp. 1400-1405, Rome, Italy, April 2007.

[8] R. Vaughan, "Massively multi-robot simulation in stage," Swarm Intelligence, vol. 2, no. 2-4, pp. 189-208, 2008.

[9] J. L. Guzmán, M. Berenguel, F. Rodríguez, and S. Dormido, "An interactive tool for mobile robot motion planning," Robotics and Autonomous Systems, vol. 56, no. 5, pp. 396-409, 2008.

[10] G. Echeverria, N. Lassabe, A. Degroote, and S. Lemaignan, "Modular open robots simulation engine: MORSE," in Proceedings of the IEEE International Conference on Robotics and Automation (ICRA '11), pp. 46-51, IEEE, Shanghai, China, May 2011.

[11] The LEGO Group, “The Lego Mindstorms home page," 2016, http://mindstorms.com.

[12] M. Casini, A. Garulli, A. Giannitrapani, and A. Vicino, "A matlab-based remote lab for multi-robot experiments," in Proceedings of the 8th IFAC Symposium on Advances in Control Education (ACE '09), pp. 162-167, Kumamoto, Japan, October 2009.

[13] M. Casini, A. Garulli, A. Giannitrapani, and A. Vicino, "A LEGO mindstorms multirobot setup in the automatic control telelab," in Proceedings of the 18th IFAC World Congress, pp. 9812-9817, Milan, Italy, 2011.

[14] M. Casini, A. Garulli, A. Giannitrapani, and A. Vicino, "A remote lab for experiments with a team of mobile robots," Sensors, vol. 14, no. 9, pp. 16486-16507, 2014.

[15] M. Casini, A. Garulli, A. Giannitrapani, and A. Vicino, "A remote lab for multi-robot experiments with virtual obstacles," in Proceedings of the 9th IFAC Symposium on Advances in Control Education (ACE '12), pp. 354-359, Nizhny Novgorod, Russia, June 2012.

[16] M. Casini, A. Garulli, A. Giannitrapani, and A. Vicino, "Remote pursuer-evader experiments with mobile robots in the automatic control telelab," in Proceedings of the 10th IFAC Symposium on Advances in Control Education (ACE '13), pp. 66-71, Sheffield, UK, August 2013.

[17] M. Casini and A. Garulli, "MARS: a Matlab simulator for mobile robotics experiments," in Proceedings of the 11th IFAC Symposium on Advances in Control Education, pp. 69-74, Bratislava, Slovakia, June 2016.

[18] J. Cortés, S. Martínez, T. Karataş, and F. Bullo, “Coverage control for mobile sensing networks," IEEE Transactions on Robotics and Automation, vol. 20, no. 2, pp. 243-255, 2004. 
[19] S. P. Lloyd, "Least squares quantization in PCM," IEEE Transactions on Information Theory, vol. 28, no. 2, pp. 129-137, 1982.

[20] Q. Du, V. Faber, and M. Gunzburger, "Centroidal Voronoi tessellations: applications and algorithms," SIAM Review, vol. 41, no. 4, pp. 637-676, 1999.

[21] H. Huang, W. Zhang, J. Ding, D. M. Stipanović, and C. J. Tomlin, "Guaranteed decentralized pursuit-evasion in the plane with multiple pursuers," in Proceedings of the 50th IEEE Conference on Decision and Control and European Control Conference (CDC-ECC '11), pp. 4835-4840, Orlando, Fla, USA, December 2011.

[22] J. A. Marshall, M. E. Broucke, and B. A. Francis, "Pursuit formations of unicycles," Automatica, vol. 42, no. 1, pp. 3-12, 2006.

[23] N. Ceccarelli, M. Di Marco, A. Garulli, and A. Giannitrapani, "Collective circular motion of multi-vehicle systems," Automatica, vol. 44, no. 12, pp. 3025-3035, 2008.

[24] D. Benedettelli, M. Casini, A. Garulli, A. Giannitrapani, and A. Vicino, "A LEGO Mindstorms experimental setup for multiagent systems," in Proceedings of the IEEE International Conference on Control Applications (CCA '09), pp. 1230-1235, Saint Petersburg, Russia, July 2009.

[25] O. Khatib, "Real-time obstacle avoidance for manipulators and mobile robots," The International Journal of Robotics Research, vol. 5, no. 1, pp. 90-98, 1986. 


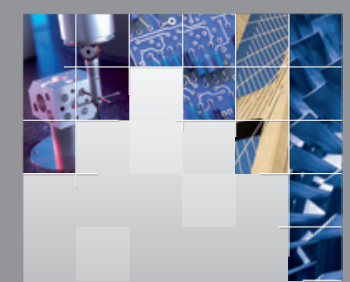

\section{Enfincering}
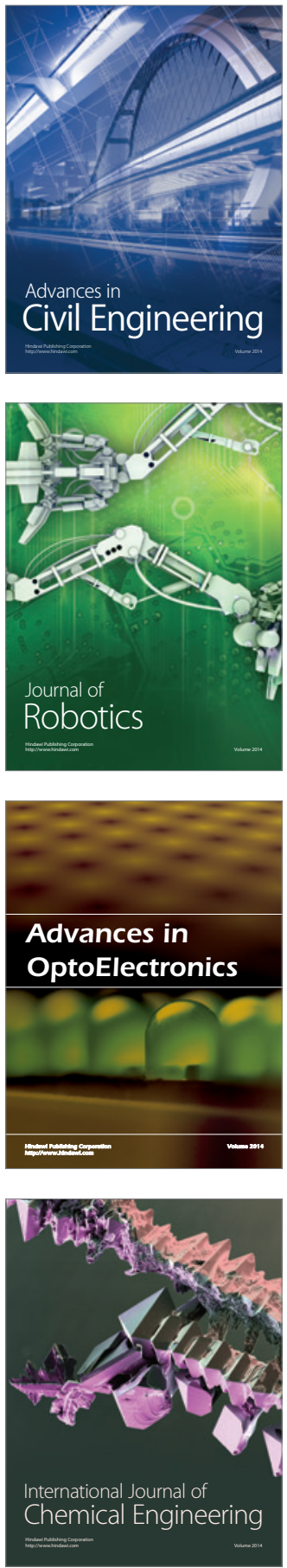

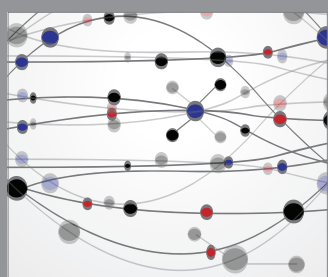

The Scientific World Journal

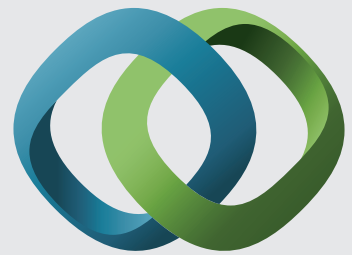

\section{Hindawi}

Submit your manuscripts at

http://www.hindawi.com
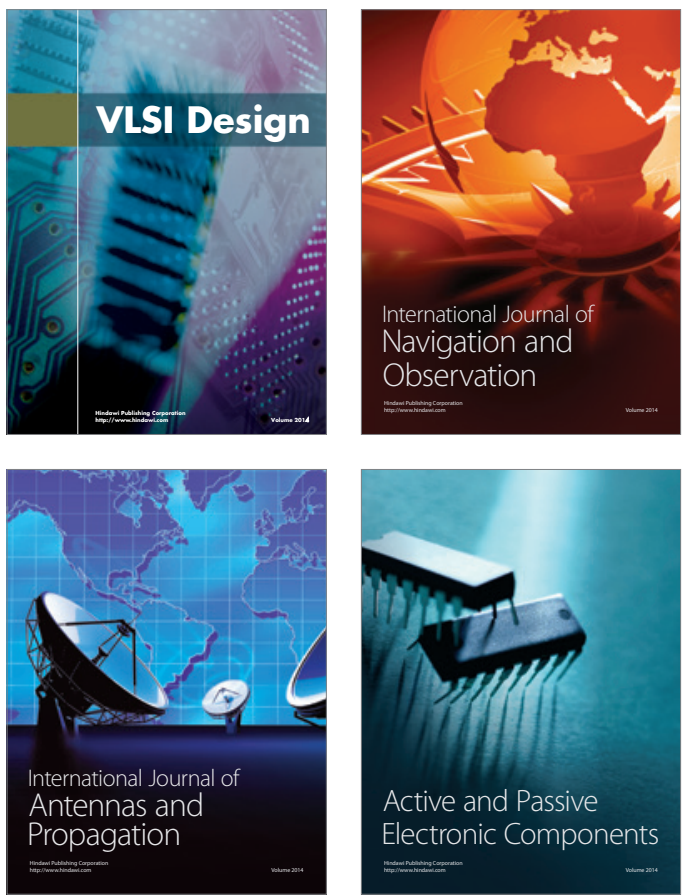
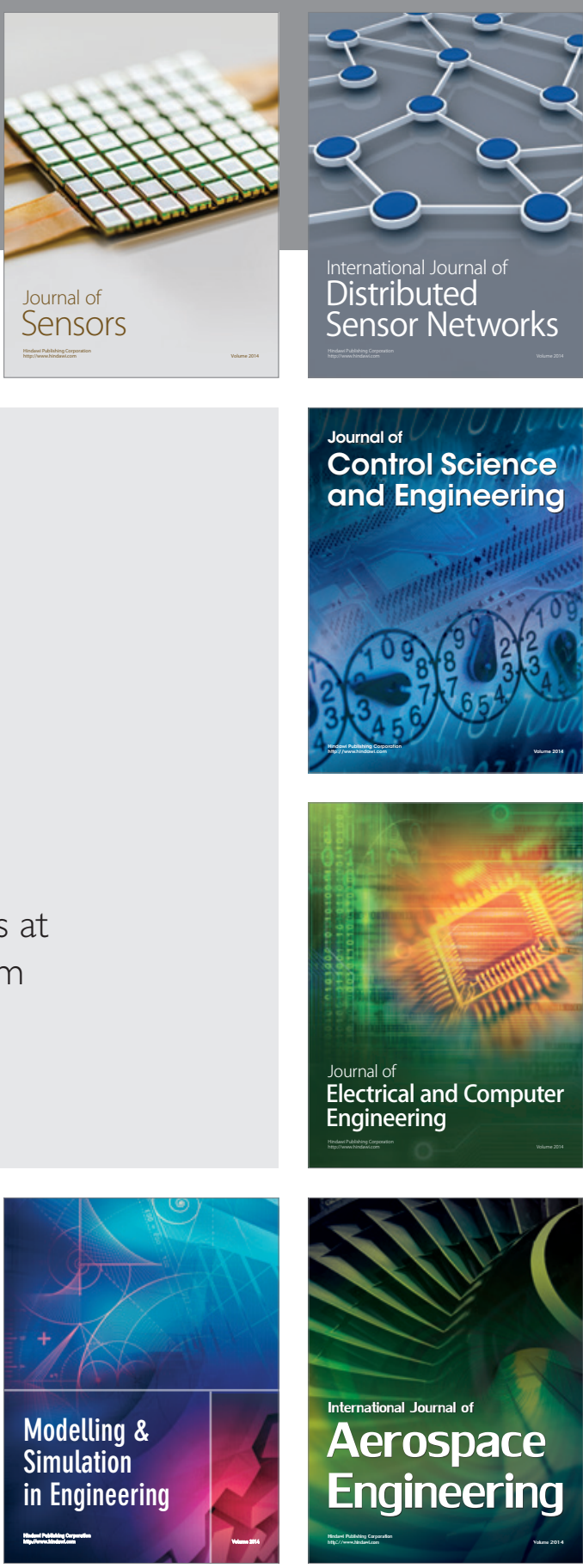

International Journal of

Distributed

Sensor Networks

Journal of

Control Science

and Engineering
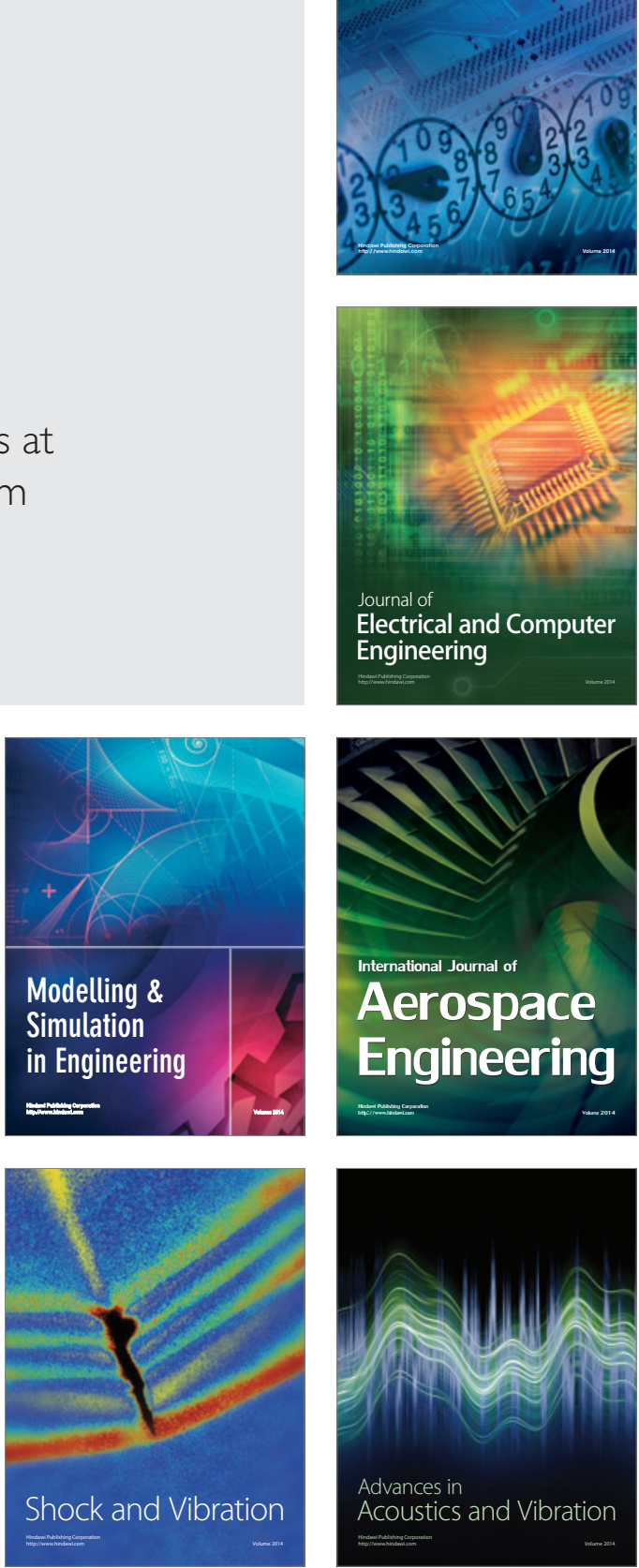